Commentary

\title{
The New Frontier in Communication Research: Why We Should Study Social Robots
}

\author{
Jochen Peter * and Rinaldo Kühne \\ Amsterdam School of Communication Research (ASCoR), University of Amsterdam, 1018 WV Amsterdam, \\ The Netherlands; E-Mails: j.peter@uva.nl (J.P.), r.j.kuhne@uva.nl (R.K.) \\ * Corresponding author
}

Submitted: 4 June 2018 | Accepted: 11 June 2018 | Published: 27 September 2018

\begin{abstract}
Social robots-robots that are made for interaction with humans-are becoming increasingly popular. In contrast to other disciplines, however, communication research has been slow in studying them. In our view, there are at least three theoretical reasons for communication researchers to deal with social robots. First, social robots challenge our notions of medium and media. Second, social robots challenge our understanding of the communication partner. Finally, social robots challenge our notions of the boundaries of communication. We therefore believe that social robots should play a more central role in communication research than it is currently the case.
\end{abstract}

\section{Keywords}

artificial intelligence; communication science; human-machine interaction; human-robot interaction; social robots

\section{Issue}

This commentary is part of the Multidisciplinary Issue of Media and Communication, edited by Epp Lauk (University of Jyväskylä, Finland) and Raul Reis (Emerson College, USA).

(C) 2018 by the authors; licensee Cogitatio (Lisbon, Portugal). This article is licensed under a Creative Commons Attribution 4.0 International License (CC BY).

\section{Introduction}

Due to ground-breaking advancements in computing, sensor technology, and artificial intelligence (e.g., Eberl, 2016), robots are nowadays increasingly designed for interaction with human beings (for a recent review see, e.g., Broadbent, 2017). These social robots integrate multiple communication modalities (e.g., vision, speech, touch) and can, once properly programmed, relate to human beings in meaningful ways (Broadbent, 2017; Fong, Nourbakhsh, \& Dautenhahn, 2003; Lee, Peng, Jin, \& Yan, 2006). As a result, social robots are already used as language tutors, as well as companions for elderly people and children with autism spectrum disorder (Cabibihan, Javed, Ang, \& Aljunied, 2013; de Graaf, Allouch, \& Klamer, 2015; Han \& Kim, 2009). Moreover, a growing number of interactive 'smart' toys, which typically rely on social-robot technology, are currently entering the market (Future of Privacy Forum \& Family Online Safety
Institute, 2016; Peter, Kühne, Barco Martelo, De Jong, \& Van Straten, in press). Finally, scholars and public commentators expect that, in the future, people will progressively encounter social robots as companions, collaborators and colleagues (e.g., Dautenhahn, 2007; Mols \& Vergunst, 2017). Against this backdrop, several observers consider social robots a key future technology (Barnatt, 2015; Ross, 2016).

While engineering sciences and robotics have been investigating social robots for some time, communication research's response to the emergence of social robots has tended to be rather slow and scattered, some notable exceptions notwithstanding (e.g., Sandry, 2015; Zhao, 2006). This is surprising because in any interaction between social robots and humans some type of communication is essential, regardless of whether this communication is verbal or non-verbal. Moreover, various scholars have recently called for more attention of communication researchers to intelligent machines (e.g., Gunkel, 
2012; Jones, 2014). In a paper published more than ten years ago and aptly titled "Humanoid Social Robots as a Medium of Communication", Zhao (2006, p. 402), for example, observed: "[The] emerging movement of social roboticization is causing a fundamental change in the meaning of social interaction and the nature of human communication in society". Focusing more broadly on semi-intelligent machines and 'smart' devices, Gunkel $(2012$, p. 2) has requested that "[c]ommunication studies...must come to terms with this development and reorient its theoretical framework". Thus, we are not the first to link social robots to communication and to propose that communication researchers should focus on human-machine communication; one of us has also requested already elsewhere that communication researchers pay attention to social robots, notably in research on young people (Peter, 2017). Based on existing research (e.g., Gunkel, 2012; Guzman, in press; Zhao, 2006), we rather believe that at least three important reasons for communication researchers to study social robots need to be (re)emphasized, especially now that the developments surrounding social robots are becoming increasingly powerful and pervasive (for an elaboration of the first two points below, see also Peter, 2017).

\section{Three Reasons to Study Social Robots}

First, social robots challenge our notions of medium and media. As Zhao (2006, p. 402) has succinctly noted, "social robots...are not a medium through which humans interact, but rather a medium with which humans interact". Social robots thus do not just function as mere transmission channels-a conceptual and theoretical problem that has been described also for computers in particular and media in general (Cathcart \& Gumpert, 1983; Gunkel, 2012). Rather, social robots transcend the role of a medium because they can be both senders and receivers and acquire the status of social actors (e.g., Gunkel, 2012; Guzman, in press). Empirical research within the computers-are-social-actors paradigm has solidly demonstrated that human beings treat computers, and media more generally, as social actors and eventually as if they were human (e.g., Reeves \& Nass, 1996). Given the vastly expanded abilities and characteristics of social robots, the idea of a medium as a communication partner thus deserves more attention (e.g., Zhao, 2006).

Second, social robots challenge our understanding of the communication partner. The vast amount of communication research-be it on interpersonal, computermediated, or mass media communication-seems to assume (at least implicitly) that communication takes place between two or more human beings (e.g., Guzman, in press). A social robot, however, can be seen as "another kind of communicative Other-who confronts human users, calls to them, and requires an appropriate response", as Gunkel (2012, p. 21) put it, referring to computers more generally. The communication partner is thus no longer human. Accordingly, social robots force us to reconsider the notion that the communicative other is typically human (Gunkel, 2012). Attention to this major shift merges partly with what has been called the 'nonhuman turn' (Grusin, 2015a). The nonhuman turn currently takes place in various fields in the social sciences and humanities that are "engaged in decentering the human in favor of a turn toward and concern for the nonhuman" (Grusin, 2015b, p. vii). We certainly do not advocate abandoning the human in communication research. Similar to others (Gunkel, 2012; Guzman, in press; Zhao, 2006), however, we do believe that an extension of our theoretical and empirical research to non-human communication partners is not only timely but will also advance the field of communication research as a whole.

Third, social robots challenge our notions of the boundaries of communication. Social robots vary in their morphology from anthropomorphic (with humanlike features), to zoomorphic (with animal features), to caricatured (similar to animation figures), to functional (with machine-like features) (Fong et al., 2003). As these robots may increasingly feature advanced and perhaps even unique skills, communication with them may go beyond what we currently know about human-human, human-animal, human-agent, or human-machine communication (Sandry, 2015). More specifically, according to some observers (e.g., van Bergen, 2016), social robots may in the future have better language and visual skills than human beings. With the advancement of social robots that are supposed to read human emotions and respond to them, such as Pepper (SoftBank Robotics), communicative possibilities may thus emerge that may exceed the boundaries of human communication (Sandry, 2015). This development may force us to confront, also in human-robot communication, what Sundar (2008) has termed the 'ftf fallacy' in reference to the relation between computer-mediated communication (CMC) and face-to-face ( $\mathrm{ftf}$ ) communication. According to Sundar (2008, p. 59), the ftf fallacy implies that "[f]tf is the gold standard, and all CMC innovations, situations, and devices are measured against this standard". Being aware of the ftf fallacy in human-robot communication means acknowledging that communication with social robots may be different from ftf communication and not necessarily comparable with it. Applied to human communication more generally, it means that human-robot communication finds its equal place next to human-human communication (e.g., Guzman, in press).

\section{Conclusion}

In summary, the emergence of social robots challenges three paradigmatic assumptions in communication research-about the medium, the communication partner and the boundaries of communication. We believe that it is crucial that communication research broadens its scope to the study of social robots in order to de- 
velop more comprehensive communication theories (for this request more generally focused on human-machine communication see, e.g., Gunkel, 2012; Guzman, in press). However, we warn researchers also against an exaggerated tech optimism, notably against overestimating the ease of doing research with current social robots (Belpaeme et al., 2013), and falling into the trap of technological determinism. Still, we are convinced that social robots should receive more attention and be given a more central position in communication research.

\section{Acknowledgements}

Work on this commentary was made possible by funding from the European Research Council (ERC) under the European Union's Horizon 2020 research and innovation programme, under grant agreement no. 682733 to the first author.

\section{Conflict of Interests}

The authors declare no conflict of interests.

\section{References}

Barnatt, C. (2015). The next big thing: From 3D printing to mining the moon. Retrieved from www.explainingthefuture.com

Belpaeme, T., Baxter, P., de Greeff, J., Kennedy, J., Read, R., Looije, R., ... Zelati, M. C. (2013). Child-robot interaction: Perspectives and challenges. In Proceedings of the 5th International Conference on Social Robotics (Vol. 8239, pp. 452-459). New York, NY: Springer.

Broadbent, E. (2017). Interactions with robots: The truths we reveal about ourselves. Annual Review of Psychology, 68(1), 627-652. https://doi.org/ 10.1146/annurev-psych-010416-043958

Cabibihan, J.-J., Javed, H., Ang, M., \& Aljunied, S. M. (2013). Why robots? A survey on the roles and benefits of social robots in the therapy of children with autism. International Journal of Social Robotics, 5(4), 593-618. https://doi.org/10.1007/s12369-0130202-2

Cathcart, R., \& Gumpert, G. (1983). Mediated interpersonal communication: Toward a new typology. Quarterly Journal of Speech, 69(3), 267-277. https://doi.org/10.1080/00335638309383654

Dautenhahn, K. (2007). Socially intelligent robots: Dimensions of human-robot interaction. Philosophical Transactions of the Royal Society of London. Series B, Biological Sciences, 362(1480), 679-704. https://doi.org/10.1098/rstb.2006.2004

de Graaf, M. M. A., Allouch, S. B., \& Klamer, T. (2015). Sharing a life with Harvey: Exploring the acceptance of and relationship-building with a social robot. Computers in Human Behavior, 43, 1-14. https://doi.org/10.1016/j.chb.2014.10.030

Eberl, U. (2016). Smarte Maschinen. Wie künstliche In- telligenz unser Leben verändert [Smart machines. How artificial intelligence changes our lives]. Munich: Hanser.

Fong, T., Nourbakhsh, I., \& Dautenhahn, K. (2003). A survey of socially interactive robots. Robotics and Autonomous Systems, 42(3/4), 143-166. https://doi.org/10.1016/S0921-8890(02)00372-X

Future of Privacy Forum, \& Family Online Safety Institute. (2016). Kids and the connected home: Privacy in the age of connected dolls, talking dinosaurs, and battling robots. Future of Privacy Forum. Retrieved from https://fpf.org/wp-content/uploads/ 2016/11/Kids-The-Connected-Home-Privacy-in-theAge-of-Connected-Dolls-Talking-Dinosaurs-and-Bat tling-Robots.pdf

Grusin, R. (Ed.). (2015a). The nonhuman turn. Minneapolis, MN: University of Minnesota Press.

Grusin, R. (2015b). Introduction. In R. Grusin (Ed.), The nonhuman turn (pp. vii-xxix). Minneapolis, MN: University of Minnesota Press.

Gunkel, D. J. (2012). Communication and artificial intelligence: Opportunities and challenges for the 21st century. Communication +1, 1(2012), 1-25.

Guzman, A. L. (in press). What is human-machine communication, anyway? In A. L. Guzman (Ed.), Humanmachine communication: Rethinking communication, technology, and ourselves. New York, NY: Peter Lang.

Han, J., \& Kim, D. (2009). r-Learning services for elementary school students with a teaching assistant robot. In Human-robot interaction (HRI) (pp. 255-256). La Jolla, CA: IEEE.

Jones, S. (2014). People, things, memory and humanmachine communication. International Journal of Media \& Cultural Politics, 10(3), 245-258. https:// doi.org/10.1386/macp.10.3.245_1

Lee, K. M., Peng, W., Jin, S.-A., \& Yan, C. (2006). Can robots manifest personality? An empirical test of personality recognition, social responses, and social presence in human-robot interaction. Journal of Communication, 56(4), 754-772. https://doi.org/ 10.1111/j.1460-2466.2006.00318.x

Mols, B., \& Vergunst, N. (2017). Hallo robot. De machine als medemens [Hello robot. The machine as a fellow human]. Amsterdam: Nieuw Amsterdam.

Peter, J. (2017). New communication technologies and young people: The case of social robots. In R. Kühne, S. E. Baumgartner, T. Koch, \& M. Hofer (Eds.), Youth and media. Current perspectives on media use and effects (pp. 203-217). Baden-Baden: Nomos.

Peter, J., Kühne, R., Barco Martelo, A., De Jong, C., \& Van Straten, C. L. (in press). Asking today the crucial questions of tomorrow: Social robots and the internet of toys. In G. Mascheroni \& D. Holloway (Eds.), The internet of toys: Practices, affordances and the political economy of children's play. Basingstoke: Palgrave Macmillan.

Reeves, B., \& Nass, C. (1996). The media equation. How people treat computers, television, and new media 
like real people and places. Cambridge: Cambridge University Press.

Ross, A. (2016). The industries of the future. New York, NY: Simon \& Schuster.

Sandry, E. (2015). Robots and communication. Basingstoke: Palgrave Macmillan.

Sundar, S. S. (2008). Self as source: Agency and customization in interactive media. In E. A. Konijn, S. Utz, M. Tanis, \& S. B. Barnes (Eds.), Mediated interpersonal communication (pp. 58-74). New York, NY:
Routledge.

van Bergen, W. (2016). De robots komen eraan! Feit en fictie over de toekomst van intelligente machines [The robots are coming! Fact and fiction about the future of intelligent machines]. Amsterdam: Business Contact.

Zhao, S. (2006). Humanoid social robots as a medium of communication. New Media \& Society, 8(3), 401-419. https://doi.org/10.1177/1461444806061951

\section{About the Authors}

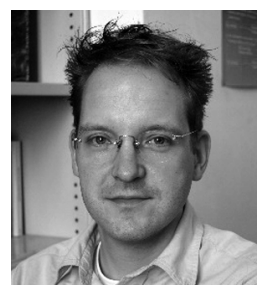

Jochen Peter (PhD, University of Amsterdam, 2003) is a Full Professor in the Amsterdam School of Communication Research (ASCOR) at the University of Amsterdam. His research deals with young people's use of new communication technologies and its consequences for their psycho-social development.

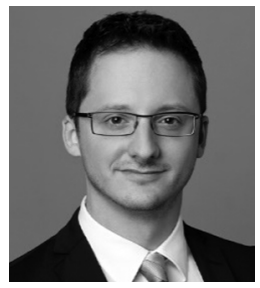

Rinaldo Kühne (PhD, University of Zürich, 2015) is an Assistant Professor in the Amsterdam School of Communication Research (ASCoR) at the University of Amsterdam. His research focuses on media effects on young people, the communication between humans and artificial agents, cognitive and emotional mechanisms of media use and effects, and methods in communication research. 